\title{
Dividend Problems in the Diffusion Model with Interest and Exponentially Distributed Observation Time
}

\author{
Cuilian Wang and Xiao Liu \\ School of Mathematics and Computer Science, Anhui Normal University, Wuhu 241000, China \\ Correspondence should be addressed to Xiao Liu; yjjatyjjat@163.com
}

Received 4 September 2013; Accepted 26 December 2013; Published 11 February 2014

Academic Editor: Bo-Qing Dong

Copyright (C) 2014 C. Wang and X. Liu. This is an open access article distributed under the Creative Commons Attribution License, which permits unrestricted use, distribution, and reproduction in any medium, provided the original work is properly cited.

\begin{abstract}
Consider dividend problems in the diffusion model with interest and exponentially distributed observation time where dividends are paid according to a barrier strategy. Assume that dividends can only be paid with a certain probability at each point of time; that is, on each observation, if the surplus exceeds the barrier level, the excess is paid as dividend. In this paper, integrodifferential equations for the moment-generating function, the $n$th moment function, and the Laplace transform of ruin time are derived; explicit expressions for the expected discounted dividends paid until ruin and the Laplace transform of ruin time are also obtained.
\end{abstract}

\section{Introduction}

The issue of maximization of the dividends paid until ruin was first proposed by De Finetti [1]. Since then the risk model in the presence of dividend payments has become a more and more popular topic in risk theory. Two recent survey papers are Avanzi [2] and Albrecher and Thonhauser's [3].

Under the dividend barrier strategy, any excess of the surplus over a given positive barrier level is immediately paid out as dividend to the shareholders of the company as long as ruin has not yet occurred. This strategy has been extensively studied by many scholars in different risk models, because it turns out that the barrier strategy is optimal among all strategies in certain situations.

The concept of randomized observation time was firstly introduced by Albrecher et al. $[4,5]$ in the classical compound Poisson risk model for the fact that insurance companies distributed dividends at discrete time points. This idea was also considered in a Brownian risk model by Albrecher et al. [6], where the waiting times between successive observation are independent random variables with a common exponential distribution. In this paper, we suppose that the surplus process of an insurance company is modelled by a Wiener process with expected increment $\mu>0$ per unit time and variance $\sigma^{2}$ per unit time and the surplus does earn interest at a constant force $\rho>0$. Under the barrier dividend strategy, we present some results on the expected discounted sum of dividends paid until ruin and the Laplace transform of ruin time.

This paper is organized as follows. In Section 2, the model we discuss in this paper is introduced. In Section 3, piecewise integrodifferential equations for the momentgenerating function, the $n$th moment function, and the Laplace transform of ruin time are derived. In Section 4, explicit expressions for the expected discounted dividends paid until ruin and the Laplace transform of ruin time are obtained.

\section{The Model}

Let $\left(\Omega, \mathfrak{F},\left\{\mathfrak{F}_{t}\right\}, P\right)$ be a filtered probability space on which all random processes and variables introduced in the following are defined. In this paper, before a dividend strategy is imposed, we assume that the surplus process of an insurance company $\{U(t) ; t \geq 0\}$ is described as

$$
\mathrm{d} U(t)=(\mu+\rho U(t)) \mathrm{d} t+\sigma \mathrm{d} W(t), \quad U(0)=u,
$$

where $u \geq 0$ is the initial surplus, $\{W(t) ; t \geq 0\}$ is a standard Brownian motion which represents diffusion, $\sigma>0$ is the diffusion coefficient, and $\rho>0$ is the interest force. 
Let $D(t)$ denote the accumulated paid dividends up to time $t$, which is an adapted càglàd (previsible, $D(t-)=$ $D(t))$ and nondecreasing process. So the controlled process is defined as

$$
\mathrm{d} X(t)=(\mu+\rho X(t)) \mathrm{d} t+\sigma \mathrm{d} W(t)-\mathrm{d} D(t), \quad X(0)=u .
$$

We assume that dividends are paid to the shareholders according to a barrier strategy with parameter $b>0$. If at a potential dividend payment time the surplus is above $b$, the excess is paid as a dividend.

Assume that the surplus process can only be observed at random times $\left\{T_{k} ; k=1,2,3, \ldots\right\}$ and the waiting times between successive observation $\left\{S_{k}=T_{k}-T_{k-1} ; k=\right.$ $1,2,3, \ldots\}\left(T_{0}=0\right)$ form a sequence of independent and identically distributed positive random variables with a common density $f_{T}(t)=\gamma e^{-\gamma t}(t>0)$. In other words, the probability that a dividend can be paid within $\mathrm{d} t$ time units is $\gamma \mathrm{d} t$ at any time. Under the barrier strategy $b$, the dividend paid at observation time $T_{k}$ is $\left(X\left(T_{k}-\right)-b\right)_{+}$, where $\left(X\left(T_{k}-\right)-b\right)_{+}=\max \left\{X\left(T_{k}-\right)-b, 0\right\}$.

Let

$$
\tau_{u, b}=\inf \{t, X(t)=0\}
$$

be the ruin time and

$$
N=\sup \left\{k \geq 0, T_{k} \leq \tau_{u, b}\right\}
$$

be the number of observation times before ruin. Assuming that dividends are discounted at a constant force of interest $\delta$ $(\delta>\rho)$, the total discounted dividends paid until ruin can be denoted as

$$
D_{u, b}=\sum_{k=1}^{N} \exp \left(-\delta T_{k}\right)\left(X\left(T_{k}-\right)-b\right)_{+}, \quad u \geq 0 .
$$

The moment-generating function is defined by

$$
M(u, y ; b)=E\left[\exp \left(y D_{u, b}\right)\right]
$$

for suitable values of $y$.

The $n$th moment function is defined by

$$
V_{n}(u ; b)=E\left[D_{u, b}^{n}\right], \quad n \in N
$$

with $V_{0}(u ; b)=1$.

The ruin probability is defined by

$$
\psi(u ; b)=P\left(\tau_{u, b}<\infty\right) .
$$

The Laplace transform of ruin time is defined by

$$
L(u ; b)=E\left[e^{-\delta \tau_{u, b}}\right] .
$$

Throughout this paper we assume that $M(u, y ; b)$, $V_{n}(u ; b)$, and $L(u ; b)$ are continuous over $u=0$, continuously differentiable over $u=b$, and twice continuously differentiable in $u \in(0, b) \cup(b, \infty)$. In addition, we assume that $M(u, y ; b)$ is continuously differentiable in $y$.

\section{Integrodifferential Equations for $M(u, y ; b), V_{n}(u ; b)$, and $L(u ; b)$}

In this section, a basic property of the expected discounted dividend payments function is given, and piecewise integrodifferential equations for the moment-generating function, the $n$th moment function, and the Laplace transform of ruin time are derived.

Clearly, $M(u, y ; b), V_{n}(u ; b)$, and $L(u ; b)$ behave differently, depending on whether their initial surplus $u$ is below or above the barrier $b$. Hence, we define

$$
\begin{gathered}
M(u, y ; b)= \begin{cases}M_{1}(u, y ; b), & u \leq b, \\
M_{2}(u, y ; b), & u>b,\end{cases} \\
V_{n}(u ; b)= \begin{cases}V_{n 1}(u ; b), & u \leq b, \\
V_{n 2}(u ; b), & u>b,\end{cases} \\
L(u ; b)= \begin{cases}L_{1}(u ; b), & u \leq b, \\
L_{2}(u ; b), & u>b .\end{cases}
\end{gathered}
$$

Proposition 1. For any $u>0$,

$$
V_{1}(u ; b) \leq \frac{\mu+\delta u}{\delta-\rho}
$$

That is to say, $V_{1}(u ; b)$ is a linear bounded function.

Proof. Let $D(t)=0$ for $t \geq \tau_{u, b}$. It is well known that the solution of stochastic differential equation (1) is

$$
U(t)=-\frac{\mu}{\rho}+e^{\rho t}\left(u+\frac{\mu}{\rho}+\int_{0}^{t} \sigma e^{-\rho s} \mathrm{~d} W(s)\right)
$$

Because $D(t) \leq X(t) \leq U(t)$, we have

$$
\begin{aligned}
V_{1}(u ; b) & =E\left[\int_{0}^{\infty} e^{-\delta t} \mathrm{~d} D(t)\right] \\
& =E\left[\delta \int_{0}^{\infty} \int_{t}^{\infty} e^{-\delta s} \mathrm{~d} s \mathrm{~d} D(t)\right] \\
& =E\left[\delta \int_{0}^{\infty} e^{-\delta s} D(s) \mathrm{d} s\right] \\
& \leq E\left[\delta \int_{0}^{\infty} e^{-\delta s} U(s) \mathrm{d} s\right] \\
& =\delta \int_{0}^{\infty} e^{-\delta s} E U(s) \mathrm{d} s \\
& =\frac{\mu+\delta u}{\delta-\rho} .
\end{aligned}
$$

The proof is completed. 
Theorem 2. The function $M(u, y ; b)$ satisfies the integrodifferential equations

$$
\begin{aligned}
& \frac{\sigma^{2}}{2} \frac{\partial^{2} M_{1}(u, y ; b)}{\partial u^{2}}+(\mu+\rho u) \frac{\partial M_{1}(u, y ; b)}{\partial u} \\
& -\delta y \frac{\partial M_{1}(u, y ; b)}{\partial y}=0, \quad 0<u<b, \\
& \frac{\sigma^{2}}{2} \frac{\partial^{2} M_{2}(u, y ; b)}{\partial u^{2}}+(\mu+\rho u) \frac{\partial M_{2}(u, y ; b)}{\partial u} \\
& -\delta y \frac{\partial M_{2}(u, y ; b)}{\partial y}-\gamma M_{2}(u, y ; b) \\
& +\gamma \exp (y(u-b)) M_{2}(b, y ; b)=0, \quad u>b
\end{aligned}
$$

with boundary conditions

$$
\begin{gathered}
M_{1}(0, y ; b)=1 ; \\
\lim _{b \rightarrow \infty} M_{1}(u, y ; b)=1 ; \\
M_{1}(b-, y ; b)=M_{2}(b+, y ; b) ; \\
\left.\frac{\partial M_{1}(u, y ; b)}{\partial u}\right|_{u=b-}=\left.\frac{\partial M_{2}(u, y ; b)}{\partial u}\right|_{u=b+} ; \\
\left.\frac{\partial^{2} M_{1}(u, y ; b)}{\partial u^{2}}\right|_{u=b-}=\left.\frac{\partial^{2} M_{2}(u, y ; b)}{\partial u^{2}}\right|_{u=b+} .
\end{gathered}
$$

Proof. When $0 \leq u<b$, consider a small time interval $(0, t]$, where $t>0$ is sufficiently small so that the surplus process will not reach $b$. In view of the strong Markov property of the surplus process $\{X(t) ; t \geq 0\}$, we have

$$
M_{1}(u, y ; b)=E\left[M_{1}\left(X(t), y e^{-\delta t} ; b\right)\right] .
$$

By Ito formula, we get

$$
\begin{aligned}
E\left[M_{1}\left(X(t), y e^{-\delta t} ; b\right)\right]= & M_{1}(u, y ; b) \\
& -\delta y \frac{\partial M_{1}(u, y ; b)}{\partial y} t \\
& +(\mu+\rho u) \frac{\partial M_{1}(u, y ; b)}{\partial u} t \\
& +\frac{\sigma^{2}}{2} \frac{\partial^{2} M_{1}(u, y ; b)}{\partial u^{2}} t .
\end{aligned}
$$

Plugging (22) into (21), dividing both sides of (21) by $t$, and letting $t \rightarrow 0$, we get (14).

When $u>b$, we separate the two possible cases as follows:

(1) no observation time occurs until $t$;

(2) an observation time occurs until $t$.
We have

$$
\begin{aligned}
M_{2}(u, y ; b)= & (1-\gamma t) E\left[M_{2}\left(X(t), y e^{-\delta t} ; b\right)\right] \\
& +\gamma t M_{2}(b, y ; b) e^{y(u-b)} .
\end{aligned}
$$

Equation (15) can be easily obtained by a similar argument.

Notice that ruin occurs immediately and no dividend is paid if $u=0$; hence (16) holds. If $b \rightarrow \infty$, there is no dividend to be paid, so (17) holds. Conditions (18) and (19) follow from the fact that $M(u, y ; b)$ is continuously differentiable. Letting $u \rightarrow b-$ in (14) and $u \rightarrow b+$ in (15), together (18) with (19), (20) holds.

Using the representation

$$
M_{i}(u, y ; b)=1+\sum_{n=1}^{\infty} \frac{y^{n}}{n !} V_{n i}(u ; b) \quad(i=1,2)
$$

and equating the coefficients of $y^{n}$ in (14), (15), and boundary conditions (16)-(20), we have the following integrodifferential equations and boundary conditions for $V_{n}(u ; b)$.

Theorem 3. The function $V_{n}(u ; b)$ satisfies the integrodifferential equations

$$
\begin{gathered}
\frac{\sigma^{2}}{2} V_{n 1}^{\prime \prime}(u ; b)+(\mu+\rho u) V_{n 1}^{\prime}(u ; b)-n \delta V_{n 1}(u ; b)=0, \\
0<u<b, \\
\frac{\sigma^{2}}{2} V_{n 2}^{\prime \prime}(u ; b)+(\mu+\rho u) V_{n 2}^{\prime}(u ; b)-(n \delta+\gamma) V_{n 2}(u ; b) \\
+\gamma \sum_{k=0}^{n} C_{n}^{k} V_{k}(b ; b)(u-b)^{n-k}, \quad u>b
\end{gathered}
$$

with boundary conditions

$$
\begin{gathered}
V_{n 1}(0 ; b)=0 ; \\
\lim _{b \rightarrow \infty} V_{n 1}(u ; b)=0 ; \\
V_{n 1}(b-; b)=V_{n 2}(b+; b) ; \\
V_{n 1}^{\prime}(b-; b)=V_{n 2}^{\prime}(b+; b) ; \\
V_{n 1}^{\prime \prime}(b-; b)=V_{n 2}^{\prime \prime}(b+; b) .
\end{gathered}
$$

Theorem 4 . The function $L(u ; b)$ satisfies the integrodifferential equations

$$
\begin{gathered}
\frac{\sigma^{2}}{2} L_{1}^{\prime \prime}(u ; b)+(\mu+\rho u) L_{1}^{\prime}(u ; b)-\delta L_{1}(u ; b)=0, \\
0<u<b, \\
\frac{\sigma^{2}}{2} L_{2}^{\prime \prime}(u ; b)+(\mu+\rho u) L_{2}^{\prime}(u ; b)-(\delta+\gamma) L_{2}(u ; b) \\
+\gamma L(b ; b)=0, \quad u>b
\end{gathered}
$$


with boundary conditions

$$
\begin{gathered}
L_{1}(0 ; b)=1 ; \\
L_{1}(b-; b)=L_{2}(b+; b) ; \\
L_{1}^{\prime}(b-; b)=L_{2}^{\prime}(b+; b) ; \\
L_{1}^{\prime \prime}(b-; b)=L_{2}^{\prime \prime}(b+; b) .
\end{gathered}
$$

Proof. The results are easily obtained by a similar argument to Theorem 2.

Theorem 5. Consider $\psi(u ; b)=1$ for any $u \in R$.

Proof. For $u \geq b$, we denote $H(u)=P\left[\inf _{0 \leq t \leq T_{1}} X(t) \leq 0\right]$; it is easy to see that $H(u)>0$. By the strong Markov property of the surplus process $\{X(t) ; t \geq 0\}$, we have

$$
\psi(u ; b)=H(u)+(1-H(u)) E\left[\psi\left(X\left(T_{1}\right) ; b\right)\right] .
$$

Since $0<X\left(T_{1}\right) \leq b \leq u$, we have $\psi\left(X\left(T_{1}\right) ; b\right) \geq \psi(u ; b)$; hence

$$
1 \geq \psi(u ; b) \geq H(u)+(1-H(u)) \psi(u ; b) .
$$

So we get $\psi(u ; b)=1$.

For $u<b, \psi(u ; b) \geq \psi(b ; b)=1$ and hence $\psi(u ; b)=1$. The proof is completed.

\section{Explicit Expressions for $V_{1}(u ; b)$ and $L(u ; b)$}

4.1. Explicit Expression for $V_{1}(u ; b)$. It is well known that the solution of (25) when $n=1$ is of the form

$$
V_{11}(u ; b)=C_{1} f_{1}(u)+C_{2} f_{2}(u)
$$

for some constants $C_{1}, C_{2}$, where

$$
\begin{gathered}
f_{1}(u)=\frac{\mu+\rho u}{\sqrt{\rho} \sigma} e^{-(\mu+\rho u)^{2} / \rho \sigma^{2}} M\left(1+\frac{\delta}{2 \rho}, \frac{3}{2} ; \frac{(\mu+\rho u)^{2}}{\rho \sigma^{2}}\right), \\
f_{2}(u)=e^{-(\mu+\rho u)^{2} / \rho \sigma^{2}} U\left(\frac{1}{2}+\frac{\delta}{2 \rho}, \frac{1}{2}, \frac{(\mu+\rho u)^{2}}{\rho \sigma^{2}}\right) .
\end{gathered}
$$

Similarly, the solution of (26) when $n=1$ is of the form

$$
V_{12}(u ; b)=C_{3} f_{3}(u)+C_{4} f_{4}(u)+A u+B
$$

for some constants $C_{3}, C_{4}, A$, and $B$, where

$$
\begin{gathered}
f_{3}(u)=\frac{\mu+\rho u}{\sqrt{\rho} \sigma} e^{-(\mu+\rho u)^{2} / \rho \sigma^{2}} M\left(1+\frac{\delta+\gamma}{2 \rho}, \frac{3}{2} ; \frac{(\mu+\rho u)^{2}}{\rho \sigma^{2}}\right), \\
f_{4}(u)=e^{-(\mu+\rho u)^{2} / \rho \sigma^{2}} U\left(\frac{1}{2}+\frac{\delta+\gamma}{2 \rho}, \frac{1}{2}, \frac{(\mu+\rho u)^{2}}{\rho \sigma^{2}}\right) .
\end{gathered}
$$

The functions $M(a, c ; x)$ and $U(a, c ; x)$ are called the confluent hypergeometric functions of the first and second kinds, respectively. We have the following properties of the two functions that

$$
\begin{gathered}
\frac{d}{d x} M(a, c ; x)=\frac{a}{c} M(a+1, c+1 ; x) ; \\
\frac{d}{d x} U(a, c ; x)=-a U(a+1, c+1 ; x) ; \\
M(a, c ; 0)=1 ; \\
\lim _{x \rightarrow \infty} M(a, c ; x)=\infty ; \\
\lim _{x \rightarrow \infty} U(a, c ; x)=0 .
\end{gathered}
$$

Since $V_{1}(u ; b)$ is a linear bounded function, it immediately follows that $C_{3}=0$.

To determine the remaining constants, we plug (41) into (26) and get

$$
\begin{gathered}
A=\frac{\gamma}{\gamma+\delta-\rho}, \\
B=\frac{\gamma\left(V_{1}(b ; b)-b\right)}{\delta+\gamma}+\frac{\gamma \mu}{(\delta+\gamma)(\delta+\gamma-\rho)} .
\end{gathered}
$$

Plugging (44) into (41) and letting $u \rightarrow b+$, we get

$$
V_{1}(b ; b)=\frac{\delta+\gamma}{\delta} C_{4} f_{4}(b)+\frac{\gamma(\mu+\rho b)}{\delta(\delta+\gamma-\rho)} .
$$

Condition (27) implies that

$$
C_{1} f_{1}(0)+C_{2} f_{2}(0)=0 ;
$$

hence $C_{2}=-\left(f_{1}(0) / f_{2}(0)\right) C_{1}$. Denoting $f(u)=f_{1}(u)-$ $\left(f_{1}(0) / f_{2}(0)\right) f_{2}(u)$, we have

$$
V_{11}(u ; b)=C_{1} f(u) .
$$

Conditions (29) and (30) give

$$
\begin{gathered}
C_{1} f(b)=\frac{\delta+\gamma}{\delta} C_{4} f_{4}(b)+\frac{\gamma(\mu+\rho b)}{\delta(\delta+\gamma-\rho)}, \\
C_{1} f^{\prime}(b)=C_{4} f_{4}^{\prime}(b)+\frac{\gamma}{\delta+\gamma-\rho} .
\end{gathered}
$$

Therefore, we now have a system of the two linear equations (48) and (49) for the two remaining constants $C_{1}, C_{4}$. A simple calculation gives

$$
\begin{aligned}
C_{1} & =\frac{\gamma}{\delta+\gamma-\rho} \frac{(\delta+\gamma) f_{4}(b)-(\mu+\rho b) f_{4}^{\prime}(b)}{(\delta+\gamma) f_{4}(b) f^{\prime}(b)-\delta f_{4}^{\prime}(b) f(b)} \\
& =\frac{\gamma}{\delta+\gamma-\rho} \frac{f_{4}^{\prime \prime}(b)}{f_{4}^{\prime \prime}(b) f^{\prime}(b)-f_{4}^{\prime}(b) f^{\prime \prime}(b)}, \\
C_{4} & =\frac{\gamma}{\delta+\gamma-\rho} \frac{\delta f(b)-(\mu+\rho b) f^{\prime}(b)}{(\delta+\gamma) f_{4}(b) f^{\prime}(b)-\delta f_{4}^{\prime}(b) f(b)} \\
& =\frac{\gamma}{\delta+\gamma-\rho} \frac{f^{\prime \prime}(b)}{f_{4}^{\prime \prime}(b) f^{\prime}(b)-f_{4}^{\prime}(b) f^{\prime \prime}(b)} .
\end{aligned}
$$


Plugging (44), (50), and (51) into (41) and (47), we obtain the explicit expression for $V_{1}(u ; b)$ that

$$
V_{11}(u ; b)=\frac{\gamma}{\delta+\gamma-\rho} \frac{f_{4}^{\prime \prime}(b)}{f_{4}^{\prime \prime}(b) f^{\prime}(b)-f_{4}^{\prime}(b) f^{\prime \prime}(b)} f(u),
$$

$$
\begin{aligned}
& V_{12}(u ; b) \\
& \quad=\frac{\gamma}{\delta+\gamma-\rho} \frac{f^{\prime \prime}(b)}{f_{4}^{\prime \prime}(b) f^{\prime}(b)-f_{4}^{\prime}(b) f^{\prime \prime}(b)} f_{4}(u)+A u+B .
\end{aligned}
$$

The optimal dividend barrier $b^{*}$ is defined as the value of $b$ which maximizes $V_{11}(u ; b)$ in $(52)$. In the following, we discuss the issue of the optimal dividend barrier. Since in (52) only the coefficient of $f(u)$ depends on the barrier level $b$, we can identify the optimal barrier $b^{*}$ which maximizes $V_{11}(u ; b)$ for a given initial capital $u$ by maximizing $C_{1}$ with respect to $b$. From now on, we regard $C_{1}$ and $C_{4}$ as the functions of $b$, and rewrite $C_{1}$ and $C_{4}$ as $C_{1}(b)$ and $C_{4}(b)$, respectively.

Proposition 6. If $(\delta+\gamma-\rho) f^{\prime \prime}(0) f_{4}^{\prime}(0)>(\delta-\rho) f^{\prime}(0) f_{4}^{\prime \prime}(0)$,

(1) there exist some $b^{*}>0$ at where $C_{1}(b)$ attains its maximum and $C_{1}^{\prime}\left(b^{*}\right)=0$;

(2) $V_{1}^{\prime}\left(b^{*} ; b^{*}\right)=1$;

(3) $\left.\left(\partial V_{12}(u ; b) / b\right)\right|_{b=b^{*}}=0$.

Proof. (1) From (50), we have

$$
\begin{aligned}
C_{1}^{\prime}(b)= & -\frac{(\delta+\gamma-\rho) C_{1}^{2}(b) f_{4}^{\prime}(b)}{\gamma\left(f_{4}^{\prime \prime}(b)\right)^{2}} \\
& \times\left[f^{\prime \prime}(b) f_{4}^{\prime \prime \prime}(b)-f^{\prime \prime \prime}(b) f_{4}^{\prime \prime}(b)\right] .
\end{aligned}
$$

Because

$$
\begin{gathered}
\frac{\sigma^{2}}{2} f_{4}^{\prime \prime}(b)=(\delta+\gamma) f_{4}(b)-(\mu+\rho b) f_{4}^{\prime}(b), \\
\frac{\sigma^{2}}{2} f^{\prime \prime}(b)=\delta f(b)-(\mu+\rho b) f^{\prime}(b),
\end{gathered}
$$

we have

$$
\begin{gathered}
\frac{\sigma^{2}}{2} f_{4}^{\prime \prime \prime}(b)=(\delta+\gamma-\rho) f_{4}^{\prime}(b)-(\mu+\rho b) f_{4}^{\prime \prime}(b), \\
\frac{\sigma^{2}}{2} f^{\prime \prime \prime}(b)=(\delta-\rho) f^{\prime}(b)-(\mu+\rho b) f^{\prime \prime}(b) .
\end{gathered}
$$

Plugging (56) into (54) yields

$$
\begin{aligned}
C_{1}^{\prime}(b)= & -\frac{2(\delta+\gamma-\rho) C_{1}^{2}(b) f_{4}^{\prime}(b)}{\gamma \sigma^{2}\left(f_{4}^{\prime \prime}(b)\right)^{2}} \\
& \times\left[(\delta+\gamma-\rho) f^{\prime \prime}(b) f_{4}^{\prime}(b)-(\delta-\rho) f^{\prime}(b) f_{4}^{\prime \prime}(b)\right] .
\end{aligned}
$$

$f_{4}^{\prime}(b)<0$ and $(\delta+\gamma-\rho) f^{\prime \prime}(0) f_{4}^{\prime}(0)>(\delta-\rho) f^{\prime}(0) f_{4}^{\prime \prime}(0)$ imply $C_{1}^{\prime}(0)>0$ and condition (28) implies $\lim _{b \rightarrow \infty} C_{1}(b)=0$. So there exist some $b^{*}>0$ at where $C_{1}(b)$ attains its maximum and $C_{1}^{\prime}\left(b^{*}\right)=0$.

(2) Since $C_{1}^{\prime}\left(b^{*}\right)=0$, we have $(\delta+\gamma-\rho) f^{\prime \prime}\left(b^{*}\right) f_{4}^{\prime}\left(b^{*}\right)-$ $(\delta-\rho) f^{\prime}\left(b^{*}\right) f_{4}^{\prime \prime}\left(b^{*}\right)=0$; hence

$$
\begin{aligned}
V_{1}^{\prime}\left(b^{*} ; b^{*}\right) & =C_{1}\left(b^{*}\right) f^{\prime}\left(b^{*}\right) \\
& =\frac{\gamma}{\delta+\gamma-\rho} \frac{f_{4}^{\prime \prime}\left(b^{*}\right) f^{\prime}\left(b^{*}\right)}{f_{4}^{\prime \prime}\left(b^{*}\right) f^{\prime}\left(b^{*}\right)-f_{4}^{\prime}\left(b^{*}\right) f^{\prime \prime}\left(b^{*}\right)} \\
& =1 .
\end{aligned}
$$

(3) Because $C_{4}^{\prime}\left(b^{*}\right)=\left(-2(\delta+\gamma-\rho) C_{4}^{2}\left(b^{*}\right) f^{\prime}\left(b^{*}\right) /\right.$ $\left.\gamma \sigma^{2}\left(f^{\prime \prime}\left(b^{*}\right)\right)^{2}\right)\left[(\delta+\gamma-\rho) f^{\prime \prime}\left(b^{*}\right) f_{4}^{\prime}\left(b^{*}\right)-(\delta-\right.$ $\left.\rho) f^{\prime}\left(b^{*}\right) f_{4}^{\prime \prime}\left(b^{*}\right)\right]=0$, we have

$$
\begin{aligned}
& \left.\frac{\partial V_{12}(u ; b)}{b}\right|_{b=b^{*}} \\
& =C_{4}^{\prime}\left(b^{*}\right) f_{4}(u) \\
& \quad+\frac{\gamma}{\delta+\gamma}\left[C_{1}^{\prime}\left(b^{*}\right) f\left(b^{*}\right)+C_{1}\left(b^{*}\right) f^{\prime}\left(b^{*}\right)-1\right]=0 .
\end{aligned}
$$

4.2. Explicit Expression for $L(u ; b)$. By a similar argument to Section 4.1, the solutions of (32) can be expressed as

$$
\begin{aligned}
L_{1}(u ; b) & =D_{1} f_{1}(u)+D_{2} f_{2}(u), \\
L_{2}(u ; b) & =D f_{4}(u)+\frac{\gamma}{\delta+\gamma} L(b ; b)
\end{aligned}
$$

for some constants $D_{1}, D_{2}$, and $D$. Letting $u \rightarrow b$ in (61), the continuity of $L(u ; b)$ over $u=b$ implies that

$$
L(b ; b)=\frac{\delta+\gamma}{\delta} D f_{4}(b) .
$$

From conditions (33)-(35), we get

$$
\begin{gathered}
D_{1} f_{1}(0)+D_{2} f_{2}(0)=1, \\
D_{1} f_{1}(b)+D_{2} f_{2}(b)=\frac{\delta+\gamma}{\delta} D f_{4}(b), \\
D_{1} f_{1}^{\prime}(b)+D_{2} f_{2}^{\prime}(b)=D f_{4}^{\prime}(b) .
\end{gathered}
$$


After some elementary algebra, we obtain

$$
\begin{aligned}
& D=\frac{\delta\left[f_{1}(b) f_{2}^{\prime}(b)-f_{1}^{\prime}(b) f_{2}(b)\right]}{\left[(\delta+\gamma) f_{4}(b) f_{2}^{\prime}(b)-\delta f_{4}^{\prime}(b) f_{2}(b)\right] f_{1}(0)+\left[\delta f_{1}(b) f_{4}^{\prime}(b)-(\delta+\gamma) f_{1}^{\prime}(b) f_{4}(b)\right] f_{2}(0)}, \\
& D_{1}=\frac{(\delta+\gamma) f_{4}(b) f_{2}^{\prime}(b)-\delta f_{4}^{\prime}(b) f_{2}(b)}{\left[(\delta+\gamma) f_{4}(b) f_{2}^{\prime}(b)-\delta f_{4}^{\prime}(b) f_{2}(b)\right] f_{1}(0)+\left[\delta f_{1}(b) f_{4}^{\prime}(b)-(\delta+\gamma) f_{1}^{\prime}(b) f_{4}(b)\right] f_{2}(0)}, \\
& D_{2}=\frac{\delta f_{1}(b) f_{4}^{\prime}(b)-(\delta+\gamma) f_{1}^{\prime}(b) f_{4}(b)}{\left[(\delta+\gamma) f_{4}(b) f_{2}^{\prime}(b)-\delta f_{4}^{\prime}(b) f_{2}(b)\right] f_{1}(0)+\left[\delta f_{1}(b) f_{4}^{\prime}(b)-(\delta+\gamma) f_{1}^{\prime}(b) f_{4}(b)\right] f_{2}(0)} .
\end{aligned}
$$

Plugging (64) into (60)-(62), we derive the explicit expression for $L(u ; b)$.

\section{Conflict of Interests}

The authors declare that there is no conflict of interests regarding the publication of this paper.

\section{Acknowledgments}

The authors would like to thank the editor and anonymous referees for their valuable comments and suggestions which lead to the improvement of this paper. This research is supported by the National Natural Science Foundation of China (11201005) and Natural Science Foundation of Anhui Province (1208085MA11).

\section{References}

[1] B. De Finetti, "Su unimpostazione alternativa dell teoria collectiva del rischio," in Transaction of the 15th International Congress of Actuaries, vol. 2, pp. 433-443, International Congress of Actuaries, New York, NY, USA, 1957.

[2] B. Avanzi, "Strategies for dividend distribution: a review," The North American Actuarial Journal, vol. 13, no. 2, pp. 217-251, 2009.

[3] H. Albrecher and S. Thonhauser, "Optimality results for dividend problems in insurance," Revista de la Real Academia de Ciencias Exactas, Fisicas y Naturales A. Matematicas, vol. 103, no. 2, pp. 295-320, 2009.

[4] H. Albrecher, E. C. K. Cheung, and S. Thonhauser, "Randomized observation periods for the compound Poisson risk model: dividends," ASTIN Bulletin, vol. 41, no. 2, pp. 645-672, 2011.

[5] H. Albrecher, E. C. K. Cheung, and S. Thonhauser, "Randomized observation periods for the compound Poisson risk model: the discounted penalty function," Scandinavian Actuarial Journal, vol. 2013, no. 6, pp. 424-452, 2013.

[6] H. Albrecher, H. U. Gerber, and E. S. W. Shiu, "The optimal dividend barrier in the Gamma-Omega model," European Actuarial Journal, vol. 1, no. 1, pp. 43-55, 2011. 


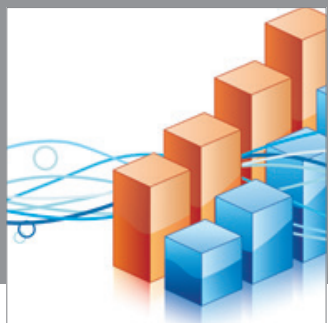

Advances in

Operations Research

mansans

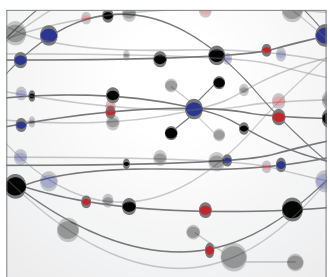

The Scientific World Journal
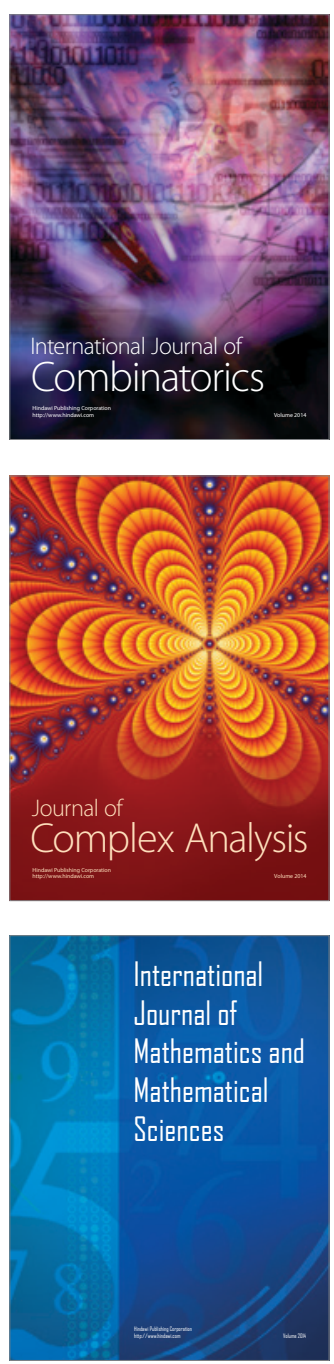
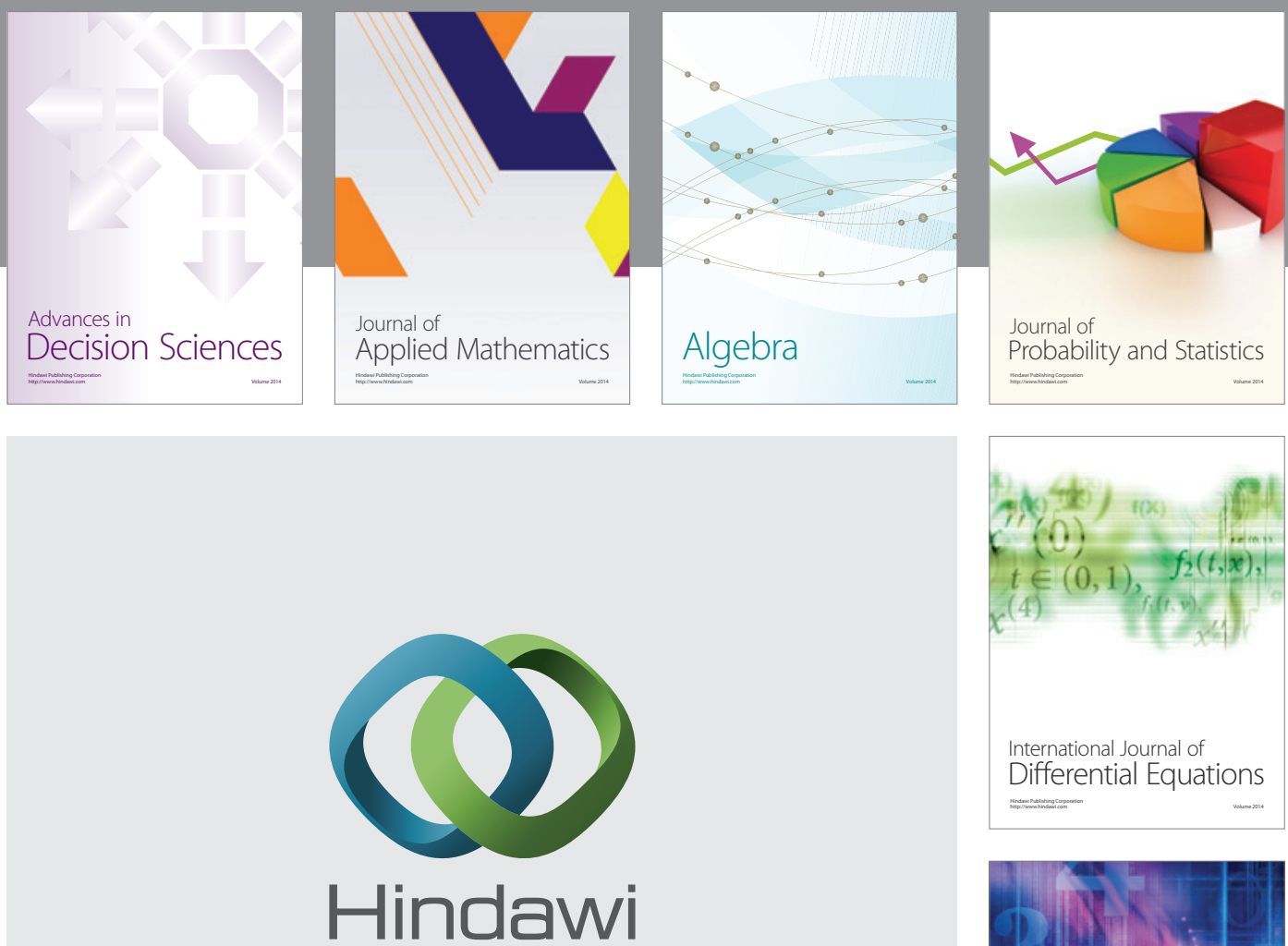

Submit your manuscripts at http://www.hindawi.com
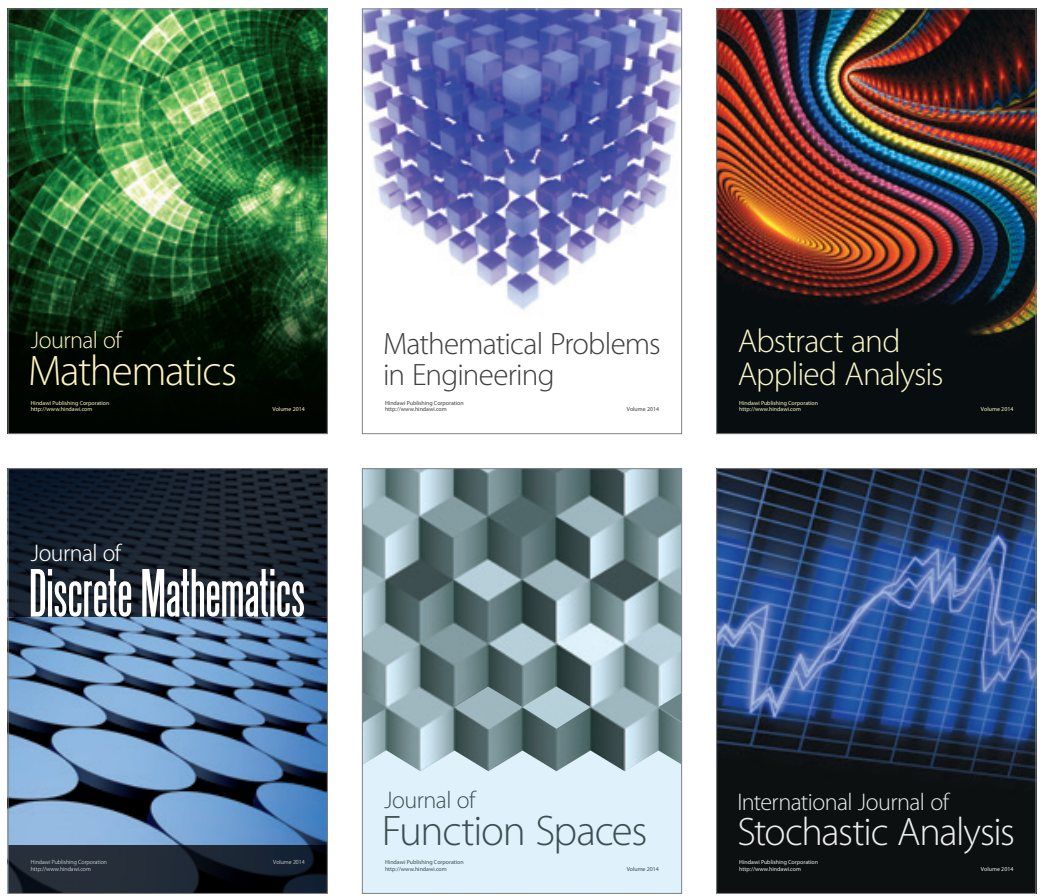

Journal of

Function Spaces

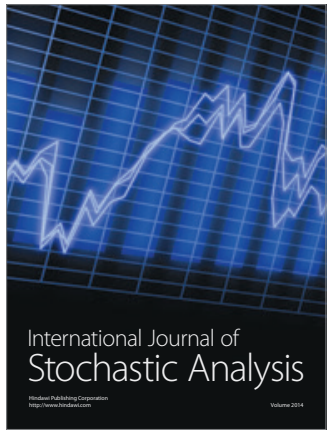

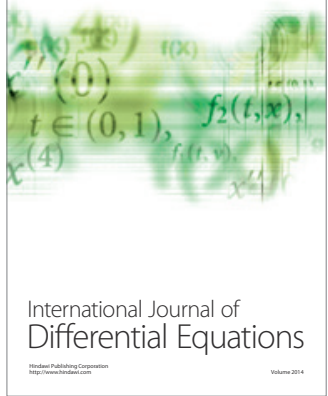
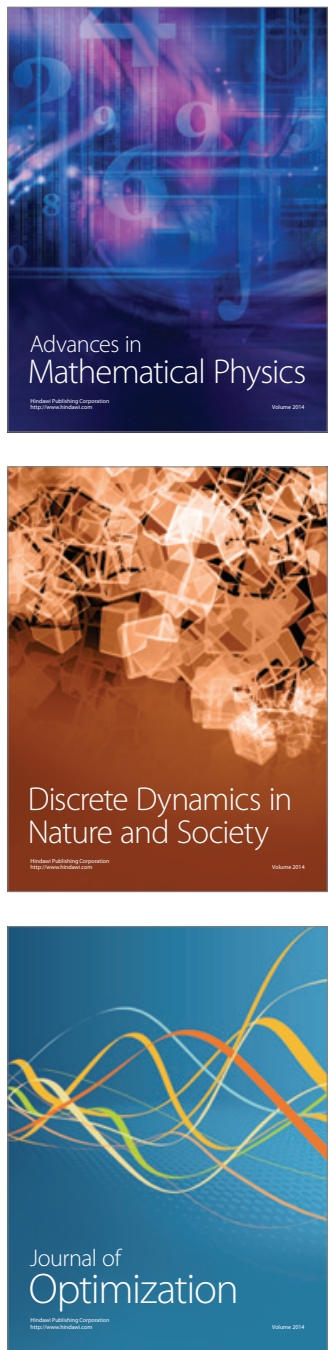